\title{
Crystal structure of pantoprazole sodium sesquihydrate Form I, $\mathrm{C}_{16} \mathrm{H}_{14} \mathrm{~F}_{2} \mathrm{~N}_{3} \mathrm{O}_{4} \mathrm{SNa}\left(\mathrm{H}_{2} \mathrm{O}\right)_{1.5}$
}

\author{
Diana Gonzalez, ${ }^{1}$ Joseph T. Golab, ${ }^{1}$ James A. Kaduk @ ${ }^{2,3, a)}$ Amy M. Gindhart, ${ }^{4}$ and \\ Thomas N. Blanton (D) \\ ${ }^{1}$ Illinois Mathematics and Science Academy, 1500 Sullivan Rd., Aurora, Illinois 60506-1000, USA \\ ${ }^{2}$ Illinois Institute of Technology, 3101 S. Dearborn St., Chicago, Illinois 60616, USA \\ ${ }^{3}$ North Central College, 131 S. Loomis St., Naperville, Illinois 60540, USA \\ ${ }^{4}$ ICDD, 12 Campus Blvd., Newtown Square, Pennsylvania 19073-3273, USA
}

(Received 16 November 2019; accepted 27 December 2019)

\begin{abstract}
The crystal structure of pantoprazole sodium sesquihydrate has been solved and refined using synchrotron X-ray powder diffraction data and optimized using density functional techniques. Pantoprazole sodium sesquihydrate crystallizes in space group Pbca (\#61) with $a=33.4862(6)$, $b=17.29311(10), c=13.55953(10) \AA, V=7852.06(14) \AA^{3}$, and $Z=16$. The crystal structure is characterized by layers parallel to the $b c$-plane. One layer contains the $\mathrm{Na}$ coordination spheres. The two independent sodium ions are trigonal bipyramidal and octahedral. The $\mathrm{NaO}_{3} \mathrm{~N}_{2}$ and $\mathrm{NaO}_{4} \mathrm{~N}_{2}$ coordination spheres share an edge to form pairs. The sodium bond valence sums are 1.17 and 1.15. The difluoromethyl groups are probably disordered. Two water molecules act as hydrogen bond donors to pyridine nitrogen atoms and sulfoxide oxygen atoms. The third water molecule participates in bifurcated hydrogen bonds, but one of its hydrogen atoms does not participate in hydrogen bonds. The powder pattern is included in the Powder Diffraction File ${ }^{\mathrm{TM}}$ as entry 00-065-1424. (C) 2020 International Centre for Diffraction Data. [doi:10.1017/S0885715620000019]
\end{abstract}

Key words: pantoprazole sodium sesquihydrate, Protonix, powder diffraction, Rietveld refinement, density functional theory

\section{INTRODUCTION}

Pantoprazole sodium sesquihydrate (brand name Protonix) is a drug classified as a proton pump inhibitor, which blocks the final step of acid production in the stomach. The reduction in acids allows for the prevention of gastroesophageal reflux disease and related symptoms. Additionally, it is used for the healing of erosive esophagitis, caused by acid damage to the esophagus. Pantoprazole is used as a short-term treatment for symptoms such as heartburn, stomach ulcers, and acid damage. The IUPAC name (CAS Registry number 164579$32-2)$ is 6-(difluoromethoxy)-2-[(3,4-dimethoxypyridin-2-yl) methylsulfinyl]-1H-benzimidazole sodium sesquihydrate. A two-dimensional molecular diagram for the pantoprazole anion is shown in Figure 1.

A low-precision powder pattern of pantoprazole sodium is contained in PDF entry 00-058-1368 (Badwan et al., 2002), and a low-precision pattern of pantoprazole sodium sesquihydrate is presented in PDF entry 00-058-1451 (Reddy et al., 2005). Several polymorphs of pantoprazole sodium are claimed in U.S. Patent 7,507,829 B2 (Finkelstein et al., 2009). Novel crystalline ketone solvates of pantoprazole sodium are disclosed in U.S. Patent Application 2005/ 024578 (Allegrini et al., 2005).

This work was carried out as a part of a project (Kaduk et al., 2014) to determine the crystal structures of largevolume commercial pharmaceuticals and include high-quality

\footnotetext{
${ }^{\text {a) }}$ Author to whom correspondence should be addressed. Electronic mail: kaduk@polycrystallography.com
}

powder diffraction data for these pharmaceuticals in the Powder Diffraction File (Gates-Rector and Blanton, 2019).

\section{EXPERIMENTAL}

Pantoprazole sodium sesquihydrate was a commercial reagent, purchased from United States Pharmacopeial Convention (USP) (Lot \# H0L207), and was used as-received.

The white powder was packed into a $1.5 \mathrm{~mm}$ diameter Kapton capillary and rotated during the measurement at $\sim 50$ Hz. The powder pattern was measured at $295 \mathrm{~K}$ at beam line 11-BM (Lee et al., 2008; Wang et al., 2008) of the Advanced Photon Source at Argonne National Laboratory using a wavelength of $0.413685 \AA$ from 0.5 to $50^{\circ} 2 \theta$ with a step size of $0.001^{\circ}$ and a counting time of $0.1 \mathrm{~s}$ per step.

The pattern was indexed on a primitive orthorhombic unit cell with $a=33.474, b=17.290, c=13.544 \AA, V=7844.5 \AA^{3}$, and $Z=16$ using Jade 9.8 (MDI, 2018). Jade, EXPO2014 (Altomare et al., 2013), FOX (Favre-Nicolin and Černý, 2002), and JANA2006 (Petricek et al., 2014) all indicated space group $\mathrm{Pbca}$ consistent with the commercial material being a racemate. A reduced cell search in the Cambridge Structural Database (Groom et al., 2016) yielded 22 hits, but no structures for pantoprazole derivatives. A pantoprazole anion was built using Spartan'18 (Wavefunction, 2018) and converted into .mol2 and .mop files using OpenBabel (O'Boyle et al., 2011). After many failures using multiple programs, the structure was finally solved using Monte Carlo-simulated annealing techniques as implemented in EXPO. Two pantoprazole anions, two $\mathrm{Na}$, and three $\mathrm{O}$ 


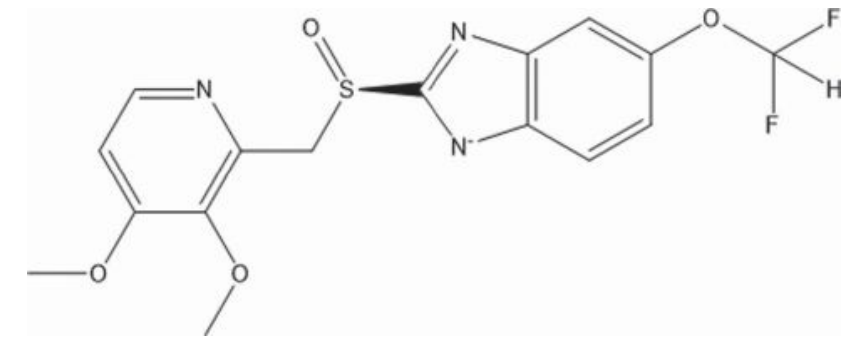

Figure 1. Molecular structure of the pantoprazole anion. (water molecules) were used as fragments. The 10 runs took $86.5 \mathrm{~h}$. One of the $\mathrm{Na}$ and two of the $\mathrm{O}$ atom positions were unreasonable (too close to pantoprazole) and removed from the model. The $\mathrm{Na}$ and two $\mathrm{O}$ were located using differentce Fourier maps. A void was indicated near the difluoromethyl groups, and a water molecule was inserted there. The TGA analysis indicated that the water content was 1.5 instead of 2.0, so this water molecule was removed. Initial hydrogen positions on the water molecules were deduced by an analysis of potential hydrogen bonding patterns.

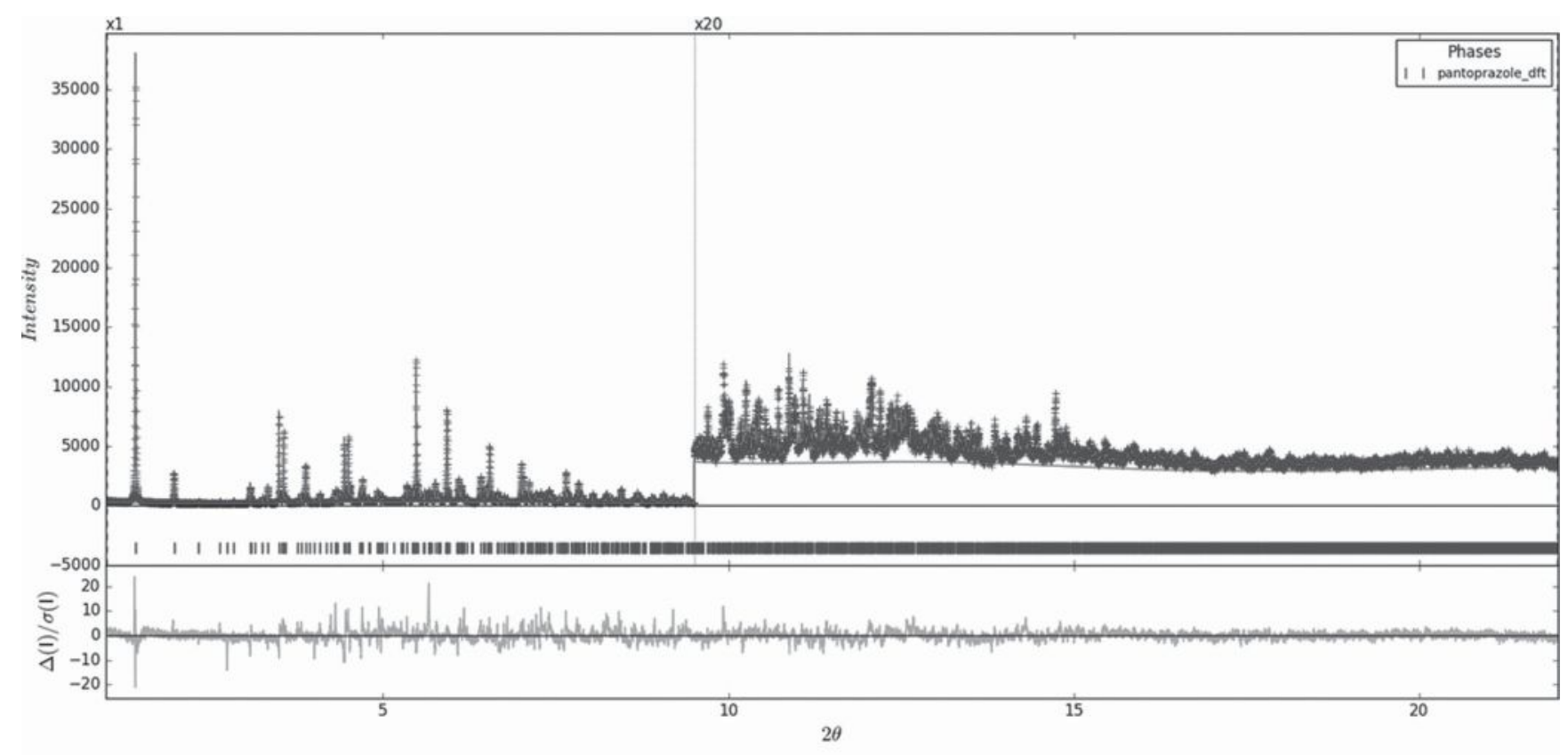

$9.5 \operatorname{deg} 20 x$

Figure 2. Rietveld plot for the refinement of pantoprazole sodium sesquihydrate. The blue crosses represent the observed data points, and the green line is the calculated pattern. The cyan curve is the normalized error plot. The vertical scale has been multiplied by a factor of $20 \times$ for $2 \theta>9.5^{\circ}$.

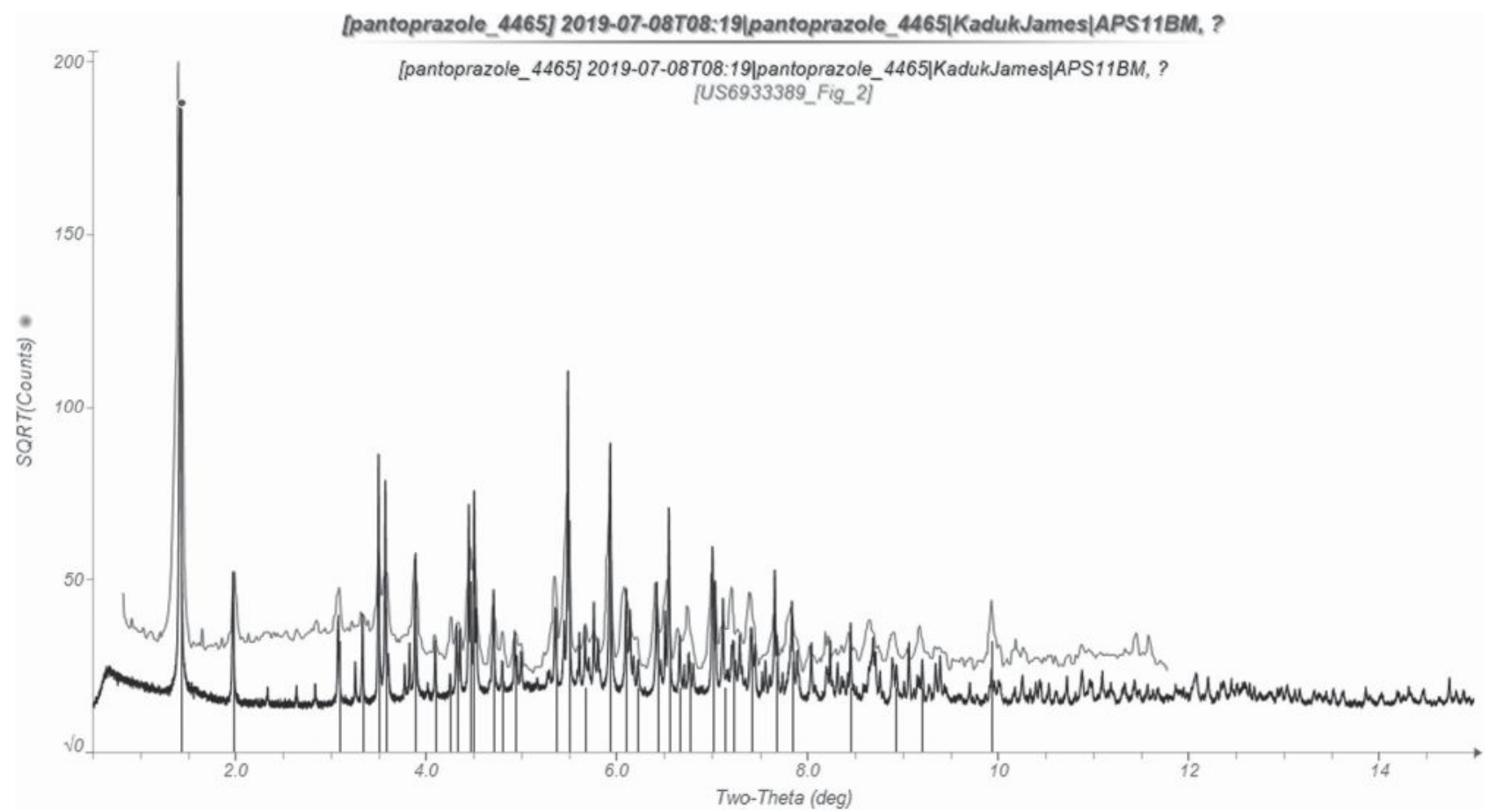

Figure 3. Comparison of the synchrotron powder pattern (blue XRD pattern) of pantoprazole sodium sesquihydrate to PDF entry 00-058-1368 (blue stick pattern) (Badwan et al., 2002) and a low-precision pattern of pantoprazole sodium sesquihydrate (green XRD pattern) (Reddy et al., 2005). 


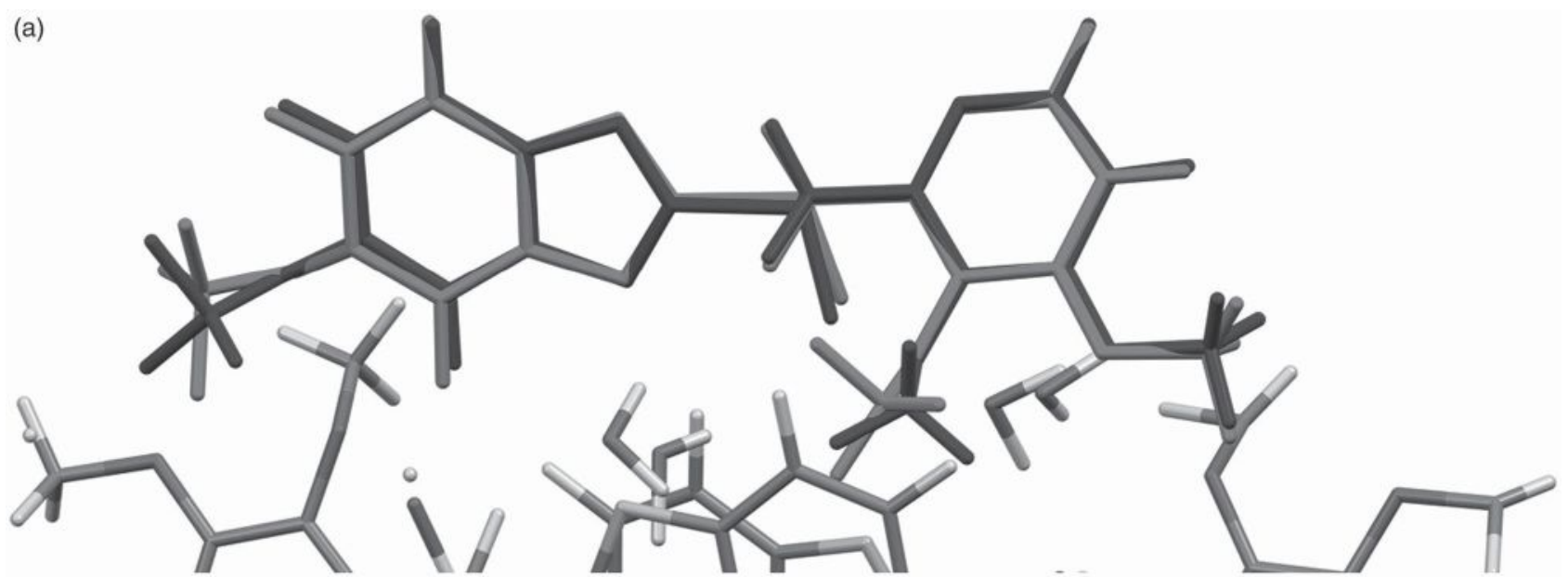

rms delta $=0.322$

(b)

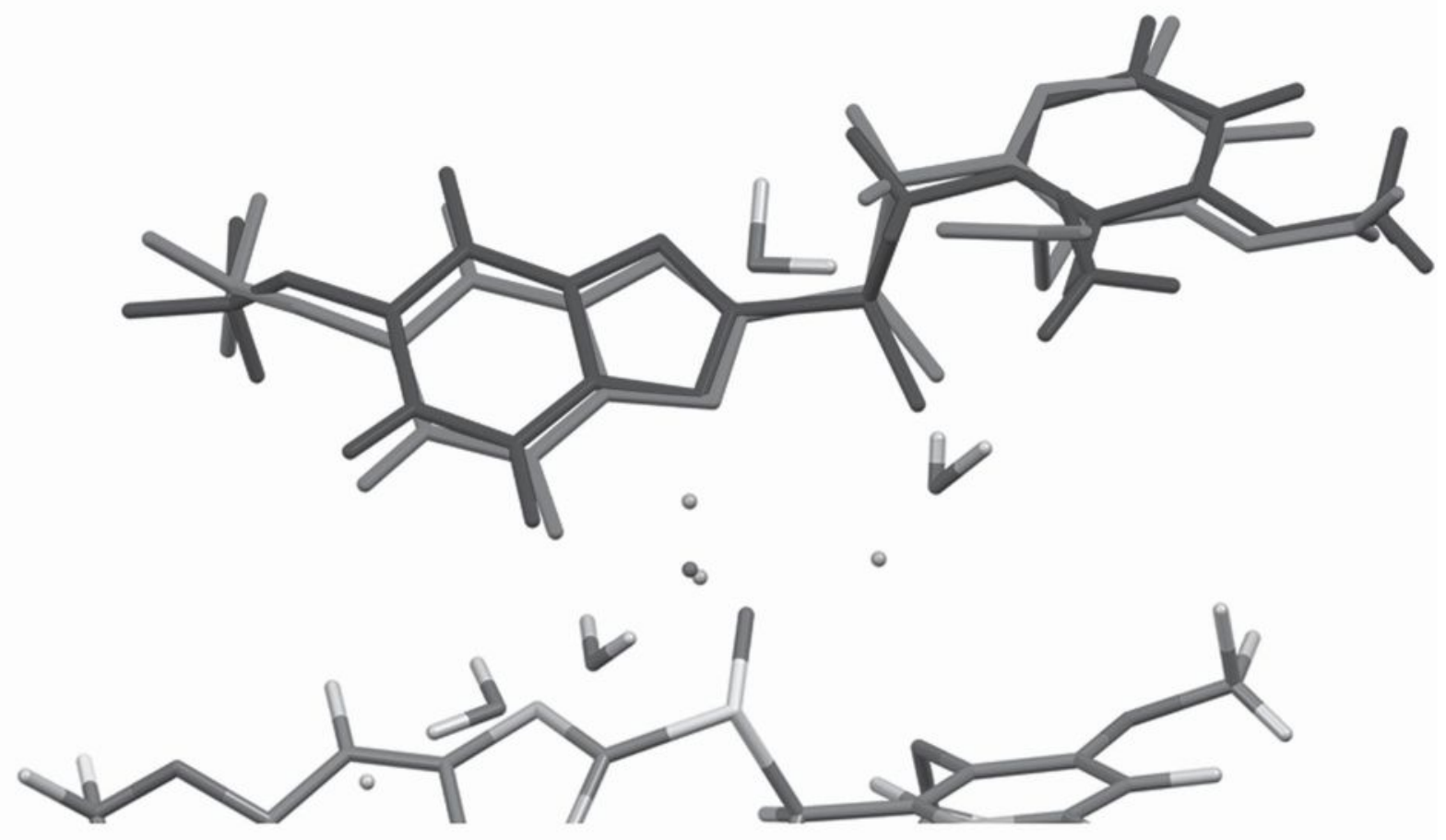

rma delta $=0.416$

Figure 4. (a) Comparison of the Rietveld-refined (red) and VASP-optimized (blue) structures of anion 1 of pantoprazole sodium sesquihydrate. The rms Cartesian displacement is $0.322 \AA$. (b) Comparison of the Rietveld-refined (red) and VASP-optimized (blue) structures of anion 2 of pantoprazole sodium sesquihydrate. The rms Cartesian displacement is $0.416 \AA$.

Rietveld refinement was carried out using GSAS-II (Toby and Von Dreele, 2013). Only the $1.0-22.0^{\circ}$ portion of the pattern was included in the refinement $\left(d_{\min }=1.084 \AA\right)$. All non-H-bond distances and angles in the anions were subjected to restraints based on a Mercury/Mogul Geometry Check (Bruno et al., 2004; Sykes et al., 2011) of the molecule. The $\mathrm{Na}-$ ligand bonds were not restrained. The pyridine and benzimidazole ring systems were restrained to be planar. The results were exported to a .csv file. The Mogul average and standard deviation for each quantity were used as the restraint parameters and were incorporated using the new feature Restraints/ Edit Restraints/Add MOGUL Restraints, which read the bond distance and angle restraints from the csv file. The restraints contributed $7.4 \%$ to the final $\chi^{2}$. The hydrogen atoms were included in calculated positions, which were recalculated during the refinement using Materials Studio (Dassault, 2018). The positions of the active hydrogen atoms were deduced by the analysis of potential hydrogen bonding patterns. The $U_{\text {iso }}$ of the non- $\mathrm{H}$ atoms of the two anions were constrained to be the same. The $U_{\text {iso }}$ of the non-H atoms were grouped by chemical similarity. A common $U_{\text {iso }}$ was refined for the atoms of the pyridine rings, the methoxy groups attached to the pyridine rings, the methylene $\mathrm{C}$ and sulfoxide $\mathrm{O}$ in the center of the anion, the $\mathrm{S}$, the atoms of the benzimidazole ring system, the difluoromethoxy group, the $\mathrm{Na}$, and the water molecule $\mathrm{O}$ atoms. The $U_{\text {iso }}$ for each hydrogen atom was constrained to be $1.3 \times$ that of the heavy atom to which it is attached. The background was modeled 


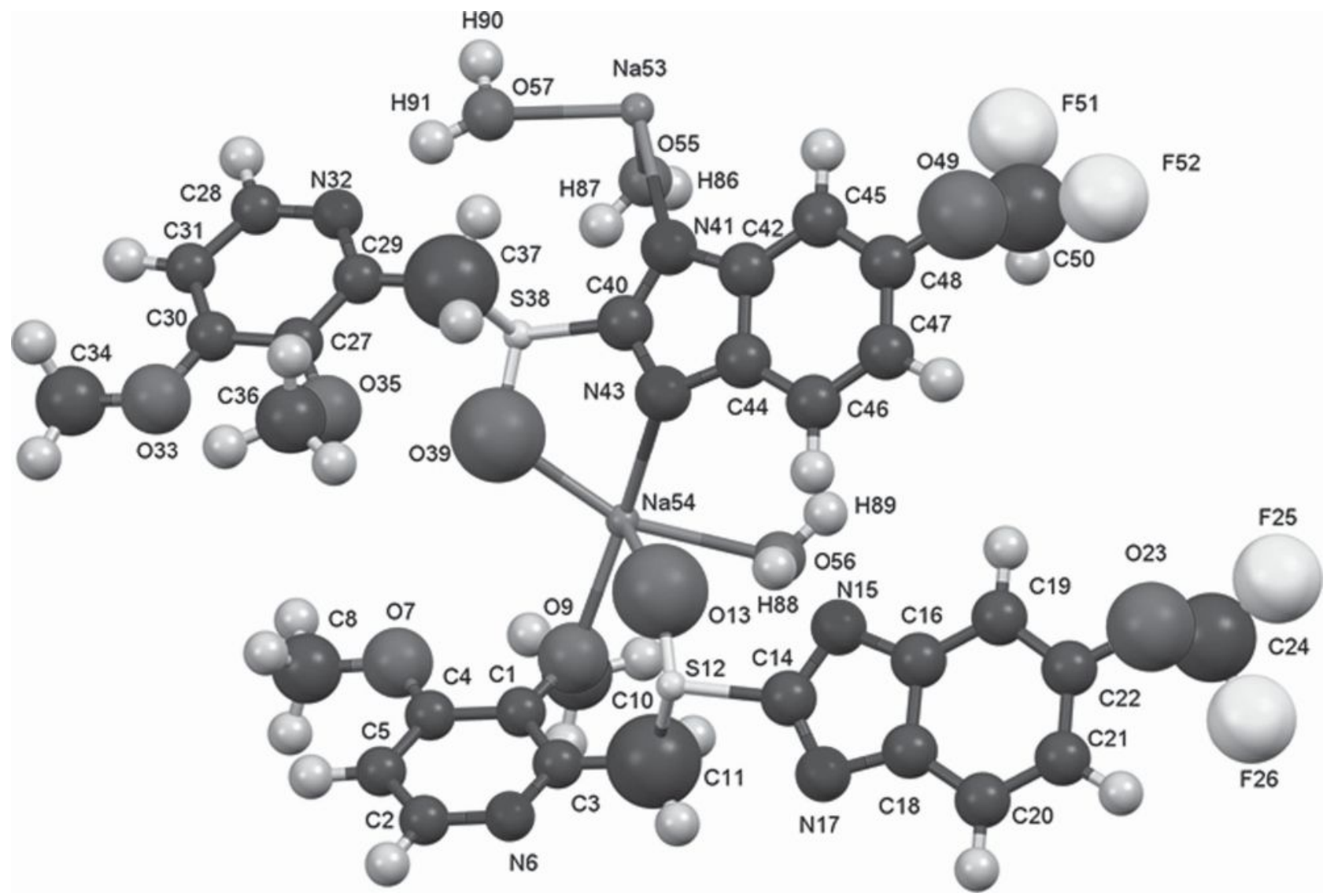

Figure 5. Asymmetric unit of pantoprazole sodium sesquihydrate, with the atom numbering. The atoms are represented by $50 \%$ probability spheroids.

using a three-term shifted Chebyshev polynomial and a sixterm diffuse scattering function to model the scattering from the Kapton capillary and any amorphous component.

The final refinement of 191 variables using 21003 observations and 138 restraints yielded the residuals $R \mathrm{wp}=0.1165$ and $\mathrm{GOF}=2.62$. The largest peak $(0.41 \AA$ from S12) and the hole $(1.29 \AA$ from S38) in the difference Fourier map were 0.55 and $-0.40(10) e \AA^{-3}$. The Rietveld plot is included in Figure 2 . The largest errors in the fit are in the intensities of some of the peaks and may represent an incomplete structural model.

A density functional geometry optimization was carried out using CRYSTAL14 (Dovesi et al., 2014). The basis sets for the $\mathrm{H}, \mathrm{C}, \mathrm{N}$, and $\mathrm{O}$ atoms were those of Gatti et al. (1994), and the basis sets for $\mathrm{S}$ and $\mathrm{Na}$ were those of Peintinger et al. (2013). The calculation was run on eight $2.1 \mathrm{GHz}$ Xeon cores (each with $6 \mathrm{~Gb}$ RAM) of a 304-core Dell Linux cluster at IIT, using eight $k$-points and the B3LYP functional, and took $\sim 30$ days.

\section{RESULTS AND DISCUSSION}

The powder pattern of the pantoprazole sodium sesquihydrate studied here matches that of U.S. Patent 6,933,389 B2 (PDF entry 00-058-1451; Reddy et al., 2005) well enough to conclude that the two materials are the same, and that our sample is representative of Form I (Figure 3).

The refined atom coordinates of pantoprazole sodium sesquihydrate and the coordinates from the density function theory (DFT) optimization are reported in the Crystallographic Information Frameworks (CIFs). The root-mean-square
Cartesian displacement of the non-hydrogen atoms in the Rietveld-refined and DFT-optimized structures of anion 1 is $0.322 \AA$ [Figure 4(a)], and the rms displacement for anion 2 is $0.416 \AA$ [Figure 4(b)]. The agreement between the refined and optimized structures is at the upper end of the range for correct structures (van de Streek and Neumann, 2014). Refinement of this very large structure with limited powder data means that this should perhaps be considered as a proposed structure. This discussion concentrates on the CRYSTAL-optimized structure. The asymmetric unit (with atom numbering) is illustrated in Figure 5, and the crystal structure is presented in Figure 6.

The crystal structure is characterized by layers parallel to the $b c$-plane [Figure 6(a)]. One layer contains the Na coordination spheres. Na53 is five-coordinate (trigonal bipyramidal), and Na54 is six-coordinate (octahedral). Na53 is coordinated to two water molecules $\mathrm{O} 55$ and O57, the sulfoxide O13, and two nitrogens of the imidazole ring (N41 and N43). Na54 is coordinated to the water molecule O56, the sulfoxide oxygens $\mathrm{O} 13$ and O39, the methoxy oxygen O9, and N17 and N43, which are the part of the imidazole rings. The $\mathrm{NaO}_{3} \mathrm{~N}_{2}$ and $\mathrm{NaO}_{4} \mathrm{~N}_{2}$ share the O13-N43 edge to form pairs [Figure 6(b)]. The bond valence sums of $\mathrm{Na} 53$ and Na54 are 1.17 and 1.15 , respectively. The Mulliken overlap populations $(\sim 0.01 e)$ indicate that $\mathrm{Na}-\mathrm{O} / \mathrm{N}$ bonds are mostly ionic, with only slight covalent character. Another layer is formed by contacts between the difluoromethyl and methoxy groups. The large displacement coefficient of the difluoromethyl groups probably means that they are disordered. Since an ordered model is necessary for the DFT calculation, we chose to let 
$\stackrel{b}{\longrightarrow}$

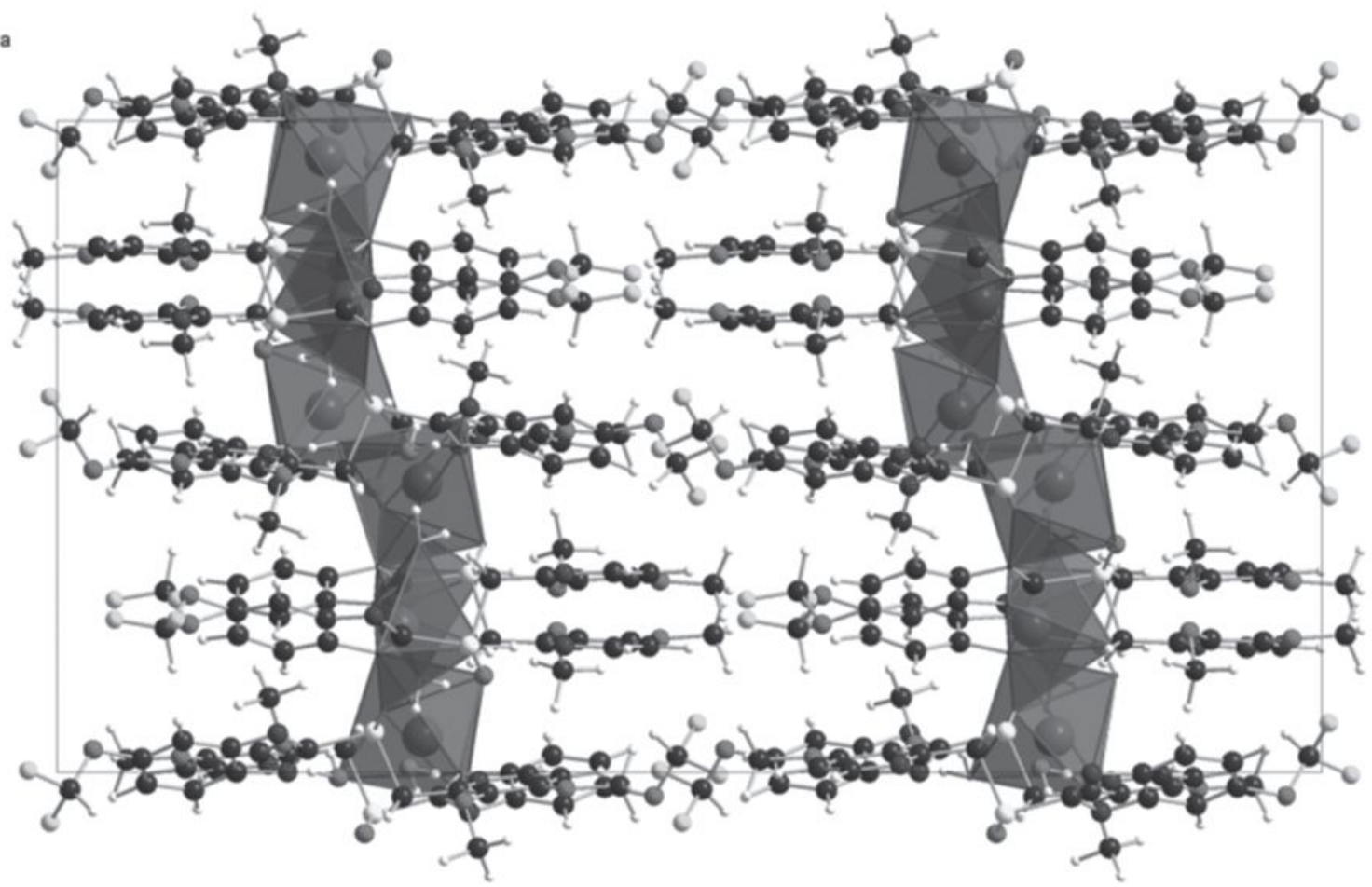

(b)
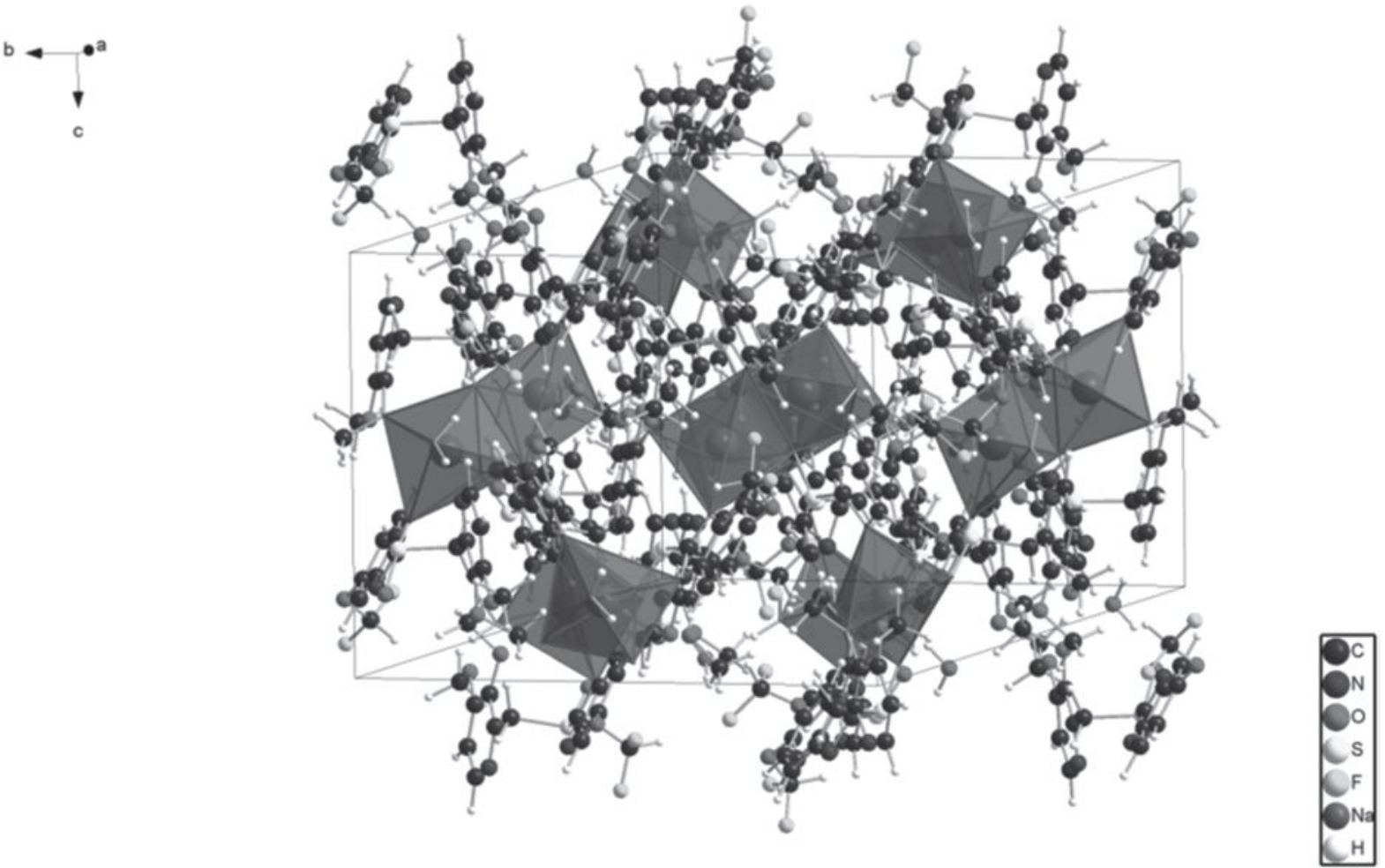

Figure 6. (a) Crystal structure of pantoprazole sodium sesquihydrate viewed down the $c$-axis. (b) Crystal structure of pantoprazole sodium sesquihydrate viewed nearly down the $a$-axis.

the $U_{\text {iso }}$ become large. We know from the molecular structure that the pantoprazole anions are ionized at the imidazole rings. Anion 1 is coordinated to Na54 only through N17 (and not $\mathrm{N} 15)$. Anion 2 is coordinated to $\mathrm{Na}$ through both $\mathrm{N} 41$ (Na53) and N43 (Na53 and Na54). The atomic charges of
$\mathrm{N} 15, \mathrm{~N} 17, \mathrm{~N} 41$, and $\mathrm{N} 43$ are $-0.520,-0.581,-0.584$, and -0.618 e, respectively. The charges of Na53 and Na54 are 0.890 and 0.885 e. The least-negative N15 is not coordinated to $\mathrm{Na}$, confirming that electrostatic contributions are important in the $\mathrm{Na}-\mathrm{N}$ bonds. 


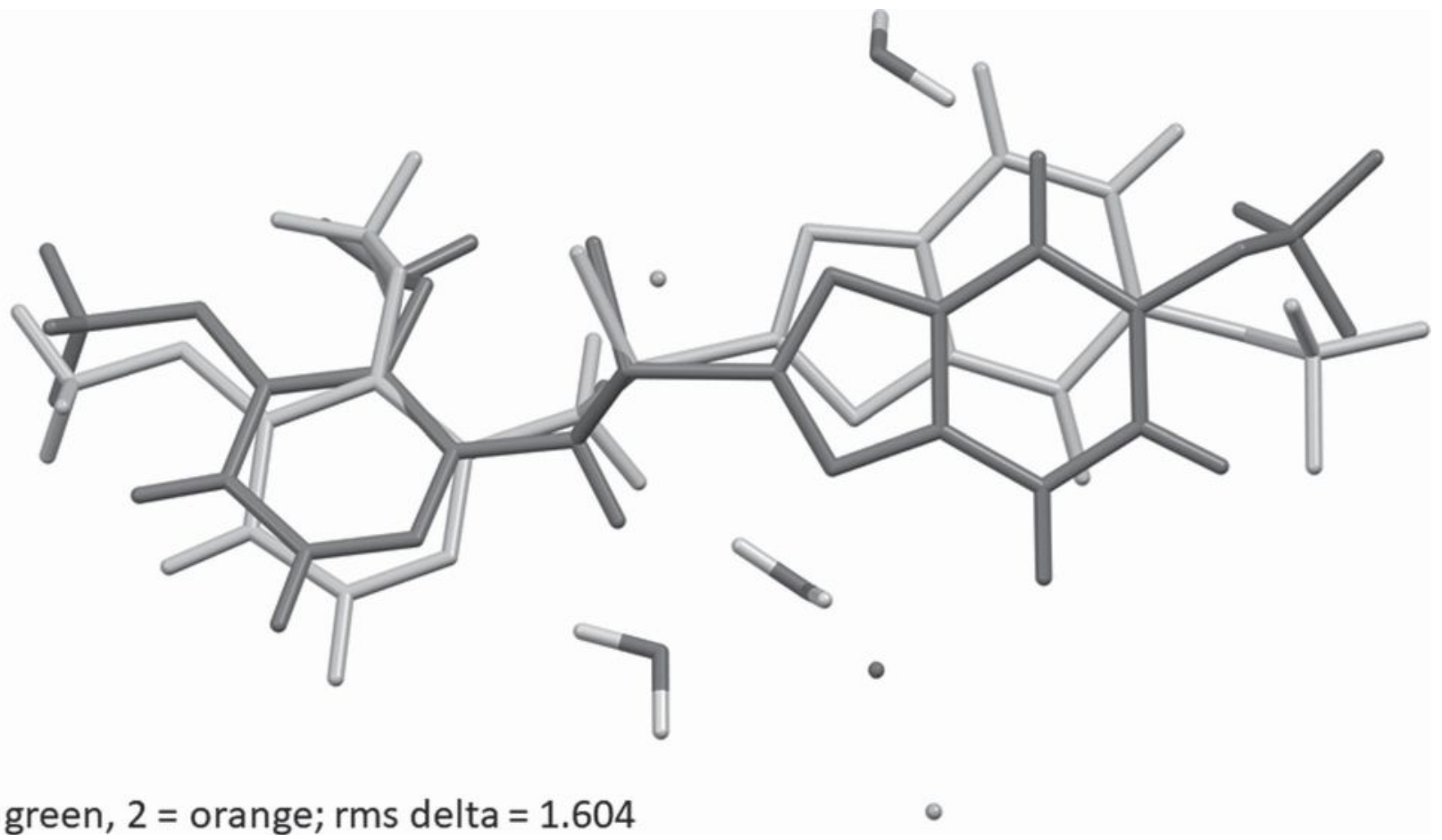

Figure 7. Comparison of the two independent anions in pantoprazole sodium sesquihydrate. Anion 1 is in green, and anion 2 is in orange.

Most of the bond distances, bond angles, and torsion angles in the anions fall within the normal ranges indicated by a Mercury Mogul Geometry check (Macrae et al., 2008). The S12-C14 and S38-C40 bond distances of 1.818 and $1.822 \AA$ are flagged as unusual (average $=1.780(10) \AA$; $Z$-scores $=3.87$ and 4.22 ), perhaps because of the small uncertainty on the average. The S38-C40-N43 angle of $118.3^{\circ}$ (average $=122.0^{\circ} ; Z$-score $\left.=4.54\right)$ is flagged as unusual. The $\mathrm{N} 43-\mathrm{C} 40-\mathrm{S} 38-\mathrm{O} 39$ torsion of $1.8^{\circ}$ lies on the tail of a broad distribution, peaking mainly from 0 to $50^{\circ}$. The N15C14-S12-O13 torsion of $13.0^{\circ}$ lies in a broad distribution, with a peak $\sim 135^{\circ}$. The N17-C14-S12-O13 torsion of $-173.8^{\circ}$ lies in a broad distribution with a peak $\sim 45^{\circ}$. All of the unusual geometrical features involve the $\mathrm{S}$ atoms, suggesting that the DFT calculation is weakest for these atoms.

The two independent anions have similar but different conformations (Figure 7). Quantum chemical geometry optimization of the pantoprazole anion (DFT/B3LYP/6-31G*/ water) using Spartan'18 (Wavefunction, 2018) indicated that the conformation of anion 2 is $64.7 \mathrm{kcal} \mathrm{mol}^{-1}$ lower in energy than anion 1 . The molecular mechanics conformational analysis indicated that the minimum-energy molecular conformation is more compact than the observed ones, with the pyridine ring rotated toward the benzimidazole ring. Intermolecular interactions, thus, are important to determine the observed conformations.

The analysis of the contributions to the total crystal energy using the Forcite module of Materials Studio (Dassault, 2018) suggests that angle and torsion distortion terms are dominant in the intramolecular deformation energy, as might be expected for anions containing a fused ring system and which are coordinated to cations. The intermolecular energy is dominated by electrostatic attractions, which in this force field-based analysis include cation coordination and hydrogen bonds. The hydrogen bonds are better analyzed using the results of the DFT calculation.

Beside coordinating to the Na cations, the water molecules act as donors in hydrogen bonds (Table I). Both $\mathrm{O}-\mathrm{H} \cdots \mathrm{O}$ and $\mathrm{O}-\mathrm{H} \cdots \mathrm{N}$ hydrogen bonds are present, and the energies of the $\mathrm{O}-\mathrm{H} \cdots \mathrm{O}$ bonds were calculated using the

TABLE I. Hydrogen bonds (CRYSTAL14) in pantoprazole sodium sesquihydrate.

\begin{tabular}{|c|c|c|c|c|c|c|}
\hline H-bond & D-H $(\AA)$ & $\mathrm{H} \cdots \mathrm{A}(\AA)$ & $\mathrm{D} \cdots \mathrm{A}(\AA)$ & $\mathrm{D}-\mathrm{H} \cdots \mathrm{A}\left({ }^{\circ}\right)$ & Overlap $(e)$ & $\left.\overline{\mathrm{E}(\mathrm{kcal} \mathrm{mol}} \mathrm{m}^{-1}\right)$ \\
\hline O57-H91 ‥N32 & 0.981 & 1.864 & 2.824 & 165.3 & 0.060 & a \\
\hline 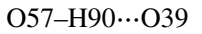 & 0.980 & 1.795 & 2.733 & 159.2 & 0.051 & 12.3 \\
\hline 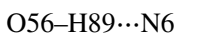 & 0.995 & 1.803 & 3.257 & 149.1 & 0.070 & a \\
\hline O56-H88‥O13 & 0.979 & 2.147 & 2.868 & 129.2 & 0.025 & 8.6 \\
\hline 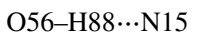 & 0.979 & 2.300 & 3.160 & 146.0 & 0.026 & a \\
\hline 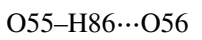 & 0.991 & 1.738 & 2.724 & 172.9 & 0.074 & 14.9 \\
\hline 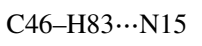 & 1.088 & 2.424 & 3.479 & 162.9 & 0.025 & a \\
\hline C37-H80‥O55 & 1.090 & 2.474 & 3.515 & 159.3 & 0.029 & a \\
\hline C20-H69...O39 & 1.082 & 2.390 & 3.091 & 121.1 & 0.018 & a \\
\hline $\mathrm{C} 11-\mathrm{H} 67 \cdots \mathrm{O} 55$ & 1.089 & 2.510 & 3.528 & 156.7 & 0.030 & a \\
\hline
\end{tabular}

${ }^{a}$ Correlation between overlap population and hydrogen bond energy not yet available for $\mathrm{C}-\mathrm{H} \cdots \mathrm{O}, \mathrm{O}-\mathrm{H} \cdots \mathrm{N}$, and $\mathrm{C}-\mathrm{H} \cdots \mathrm{N}$ hydrogen bonds. 


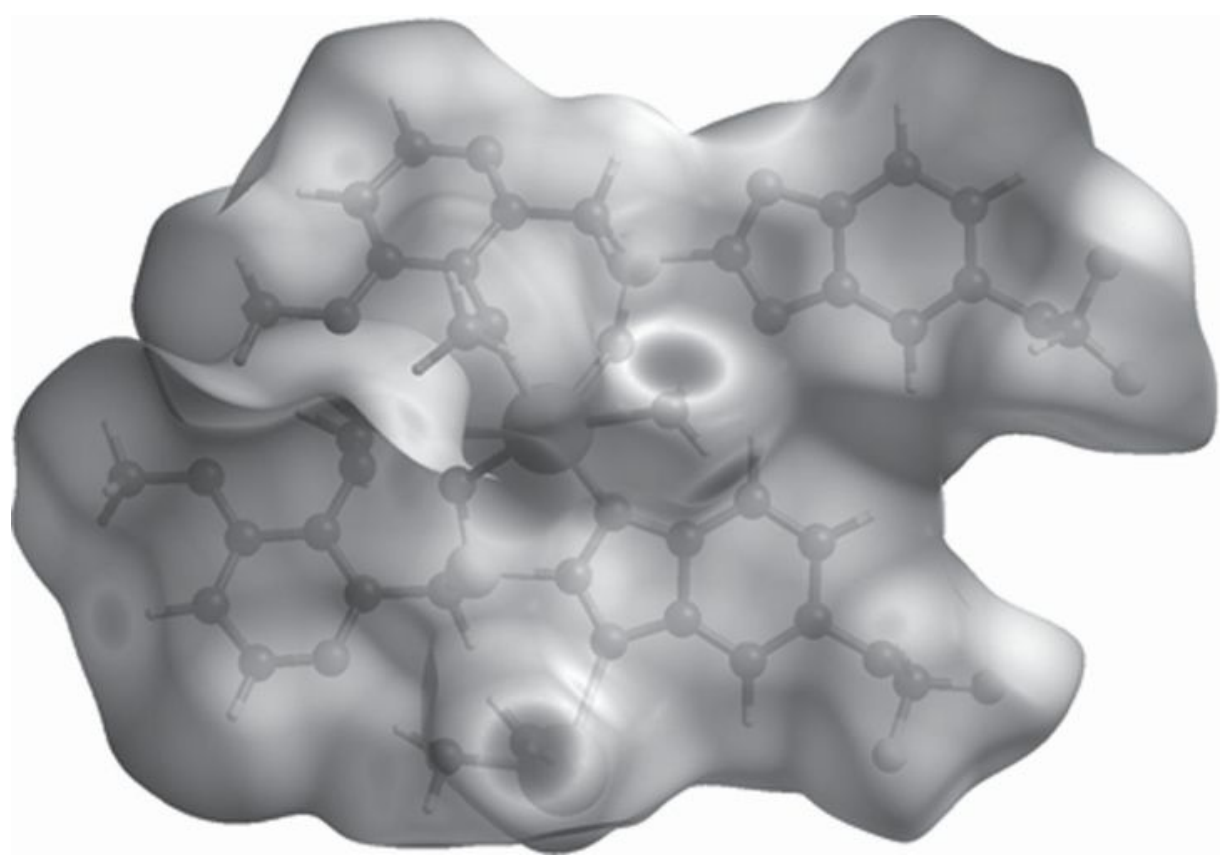

Figure 8. Hirshfeld surface of pantoprazole sodium sesquihydrate. Intermolecular contacts longer than the sums of the van der Waals radii are colored blue, and contacts shorter than the sums of the radii are colored red. Contacts equal to the sums of radii are white.

correlation of Rammohan and Kaduk (2018). Both O57 and O56 act as donors to pyridine nitrogens and sulfoxide oxygens. H88, which is on O55, participates in bifurcated hydrogen bonds to $\mathrm{O} 13$ and $\mathrm{N} 15$. H87 does not act as a donor, but $\mathrm{O} 55$ acts as an acceptor in two $\mathrm{C}-\mathrm{H} \cdots \mathrm{O}$ hydrogen bonds.

The volume enclosed by the Hirshfeld surface (Figure 8; Hirshfeld, 1977; Turner et al., 2017) is $967.66 \AA^{3}, 98.59 \%$ of $1 / 8$ the unit cell volume. The packing density is thus normal. All of the significant close contacts (red in Figure 8) involve the hydrogen bonds. The volume/non-hydrogen atom is $18.5 \AA^{3}$.

The Bravais-Friedel-Donnay-Harker (Bravais, 1866; Friedel, 1907; Donnay and Harker, 1937) morphology suggests that we might expect platy morphology for pantoprazole sodium sesquihydrate, with $\{200\}$ as the principal faces. A second-order spherical harmonics model was included in the refinement. The texture index was 1.002 , indicating that the preferred orientation was not significant in the rotated capillary specimen. The powder pattern of pantoprazole sodium sesquihydrate from a Le Bail extraction of this synchrotron data set is included in the Powder Diffraction File ${ }^{\mathrm{TM}}$ as entry 00-65-1424.

\section{DEPOSITED DATA}

The CIF files containing the results of the Rietveld refinement (including the raw data) and the DFT geometry optimization were deposited with the ICDD. The data can be requested atinfo@icdd.com

\section{ACKNOWLEDGEMENTS}

Use of the Advanced Photon Source at Argonne National Laboratory was supported by the U.S. Department of Energy, Office of Science, and Office of Basic Energy Sciences, under Contract No. DE-AC02-06CH11357. We thank Lynn Ribaud and Saul Lapidus for their assistance in the data collection, and Andrey Rogachev for the use of computing resources at IIT.

\section{FUNDING INFORMATION}

This work was partially supported by the International Centre for Diffraction Data.

\section{CONFLICTS OF INTEREST}

The authors have no conflicts of interest to declare.

Allegrini, P., Ventimiglia,, G., Sommei, A., and Castaldi, G. (2005). "Polymorphs of pantoprazole sodium salt and process for the preparation thereof," U. S. Patent Application 2005/024578 A1.

Altomare, A., Cuocci, C., Giacovazzo, C., Moliterni, A., Rizzi, R., Corriero, N., and Falcicchio, A. (2013). "EXPO2013: a kit of tools for phasing crystal structures from powder data," J. Appl. Crystallogr. 46, 1231-1235.

Badwan, A., Nabulsi, L., Al Omari, M., Daraghmeh, N., Ashour, M., Abdoh, A., and Jaber, A. (2002). "Pantoprazole sodium," Anal. Profiles Drug Subst. Excipients. 29, 213-259.

Bravais, A. (1866). Etudes Cristallographiques ((Gauthier Villars, Paris.

Bruno, I. J., Cole, J. C., Kessler, M., Luo, J., Motherwell, W. D. S., Purkis, L. H., Smith, B. R., Taylor, R., Cooper, R. I., Harris, S. E., and Orpen, A. G. (2004). "Retrieval of crystallographically-derived molecular geometry information," J. Chem. Inf. Sci. 44, 2133-2144.

Dassault Systèmes (2018). Materials Studio 2019 (BIOVIA, San Diego, CA).

Donnay, J. D. H., and Harker, D. (1937). "A new law of crystal morphology extending the law of Bravais," Am. Min. 22, 446-447.

Dovesi, R., Orlando, R., Erba, A., Zicovich-Wilson, C. M., Civalleri, B., Casassa, S., Maschio, L., Ferrabone, M., De La Pierre, M., D-Arco, P., Noël, Y., Causà, M., and Kirtman, B. (2014). "CRYSTAL14: a program for the ab initio investigation of crystalline solids," Int. J. Quantum Chem. 114, 1287-1317.

Favre-Nicolin, V., and Černý, R. (2002). "FOX, 'free objects for crystallography': a modular approach to ab initio structure determination from powder diffraction," J. Appl. Crystallogr. 35, 734-743.

Finkelstein, N., Krochmal, B., Wizel, S., and Braude, V. (2009). "Solid states of pantoprazole sodium, processes for preparing them and processes for 
preparing known pantoprazole sodium hydrates," U.S. Patent 7,507,829 B2.

Friedel, G. (1907). "Etudes sur la loi de Bravais," Bull. Soc. Fr. Mineral. 30, 326-455.

Gates-Rector, S., and Blanton, T. (2019). "The powder diffraction file: a quality materials characterization database," Powder Diffr. 34(4), 352-360.

Gatti, C., Saunders, V. R., and Roetti, C. (1994). "Crystal-field effects on the topological properties of the electron-density in molecular crystals - the case of urea," J. Chem. Phys. 101, 10686-10696.

Groom, C. R., Bruno, I. J., Lightfoot, M. P., and Ward, S. C. (2016). "The Cambridge Structural Database," Acta Crystallogr. Sect. B: Struct. Sci., Cryst. Eng. Mater. 72, 171-179.

Hirshfeld, F. L. (1977). "Bonded-atom fragments for describing molecular charge densities," Theor. Chem. Acta. 44, 129-138.

Kaduk, J. A., Crowder, C. E., Zhong, K., Fawcett, T. G., and Suchomel, M. R. (2014). "Crystal structure of atomoxetine hydrochloride (Strattera), $\mathrm{C}_{17} \mathrm{H}_{22} \mathrm{NOCl}$," Powder Diffr. 29(3), 269-273.

Lee, P. L., Shu, D., Ramanathan, M., Preissner, C., Wang, J., Beno, M. A., Von Dreele, R. B., Ribaud, L., Kurtz, C., Antao, S. M., Jiao, X., and Toby, B. H. (2008). "A twelve-analyzer detector system for highresolution powder diffraction," J. Synch. Rad. 15(5), 427-432.

Macrae, C. F., Bruno, I. J., Chisholm, J. A., Edington, P. R., McCabe, P., Pidcock, E., Rodriguez-Monge, L., Taylor, R., van de Streek, J., and Wood, P. A. (2008). "Mercury CSD 2.0 - new features for the visualization and investigation of crystal structures," J. Appl. Crystallogr. 41, 466-470.

MDI (2018). Jade 9.8 (Materials Data Inc., Livermore, CA).

O'Boyle, N., Banck, M., James, C. A., Morley, C., Vandermeersch, T., and Hutchison, G. R. (2011). "Open Babel: an open chemical toolbox," J. Chem. Informatics. 3, 33.
Peintinger, M. F., Vilela Oliveira, D., and Bredow, T. (2013). "Consistent Gaussian basis sets of triple-zeta valence with polarization quality for solid-state calculations," J. Comput. Chem. 34, 451-459.

Petricek, V., Dusek, M., and Palatinus, L. (2014). "Crystallographic computing system JANA2006: general features,” Z. Kristallogr. 229, 345-352.

Rammohan, A., and Kaduk, J. A. (2018). "Crystal structures of alkali metal (Group 1) citrate salts," Acta Crystallogr. Sect. B: Cryst. Eng. Mater. 74, 239-252.

Reddy, M., Eswaraiah, S., Mathad, V., Anilkumar, P., Chandrashekar, E., and Shanmugam, G. (2005). "Process for preparation of crystalline form-I of pantoprazole sodium sesquihydrate," U.S. Patent 6,933,389 B2.

Sykes, R. A., McCabe, P., Allen, F. H., Battle, G. M., Bruno, I. J., and Wood, P. A. (2011). "New software for statistical analysis of Cambridge Structural Database data,” J. Appl. Crystallogr. 44, 882-886.

Toby, B. H., and Von Dreele, R. B. (2013). "GSAS II: the genesis of a modern open source all purpose crystallography software package," J. Appl. Crystallogr. 46, 544-549.

Turner, M. J., McKinnon, J. J., Wolff, S. K., Grimwood, D. J., Spackman, P. R., Jayatilaka, D., and Spackman, M. A. (2017). CrystalExplorer 17 (University of Western Australia). Available at: http://hirshfeldsurface.net.

van de Streek, J., and Neumann, M. A. (2014). "Validation of molecular crystal structures from powder diffraction data with dispersion-corrected density functional theory (DFT-D)," Acta Crystallogr. Sect. B: Struct. Sci., Cryst. Eng. Mater. 70(6), 1020-1032.

Wang, J., Toby, B. H., Lee, P. L., Ribaud, L., Antao, S. M., Kurtz, C., Ramanathan, M., Von Dreele, R. B., and Beno, M. A. (2008). "A dedicated powder diffraction beamline at the advanced photon source: commissioning and early operational results," Rev. Sci. Instrum. 79, 085105.

Wavefunction, Inc. (2018). Spartan'18 Version 1.2.0 (Wavefunction Inc., Irvine, CA). 